\title{
Oesophagogastrectomy using the end-to-end anastomosing stapler
}

\author{
B FABRI, RJ DONNELLY \\ From the Cardiothoracic Surgical Centre, Broadgreen Hospital, Liverpool
}

\begin{abstract}
The use of a mechanical instrument for accomplishing end-to-side oesophagogastrostomy is described. We report our experience with 30 consecutive patients undergoing oesophagogastrectomy-26 for malignant disease and four for benign peptic stricture. There were two immediate postoperative deaths. One patient developed a chylothorax and died six weeks after operation. The instrument failed to function satisfactorily on one occasion. There were no anastomotic leaks.
\end{abstract}

The use of mechanical stapling devices in surgery has been reported extensively over the past 30 years. ${ }^{1-5}$ Instruments capable of accomplishing a circular anastomosis are a more recent development. ${ }^{6-8}$ The stapled anastomosis is claimed to be safer than conventional hand-sewn methods and therefore to represent an important advance in reducing morbidity and mortality associated with

Address for reprint requests: Mr RJ Donnelly, Cardiothoracic Surgical Centre, Broadgreen Hospital, Thomas Drive, Liverpool L14 3LB. oesophageal resection. It also provides a time-saving factor, but this is probably less important than the reduction in the incidence of suture line breakdown. In this paper we present our results using the Auto Suture End-to-End anastomosing (EEA) instrument (fig 1) in 30 patients undergoing oesophagogastrectomy.

\section{Patients and method}

Between January and December 1980, oesophagogastrectomy was performed on 30 consecutive

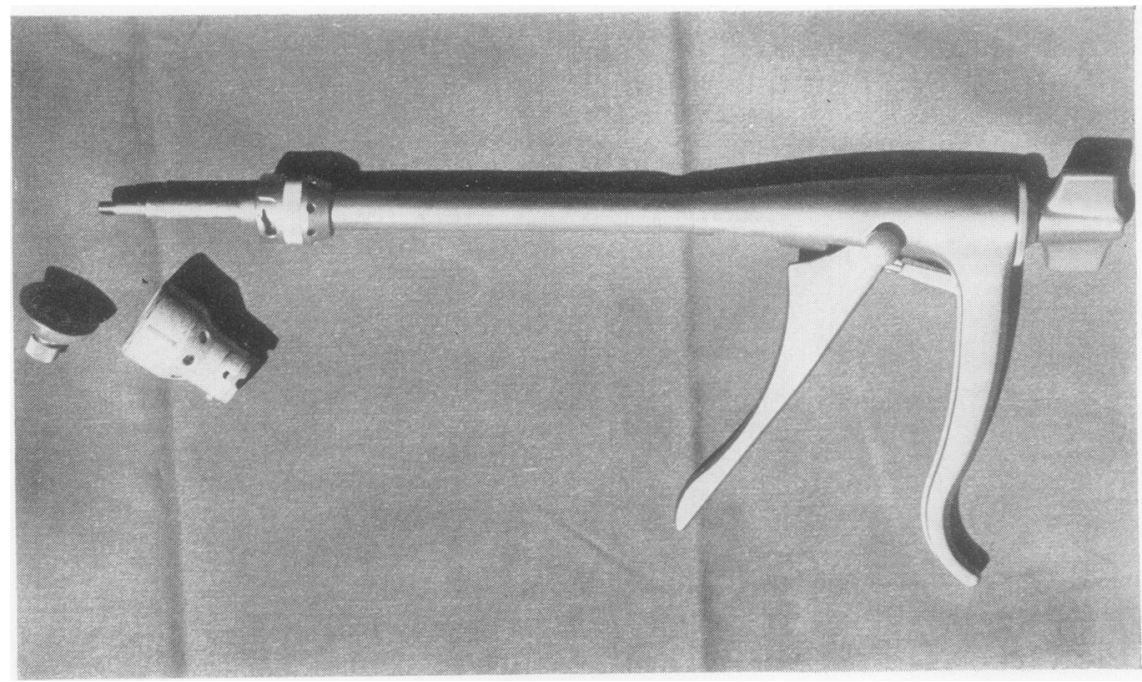

Fig 1 Autosuture end-to-end anastomosing (EEA) stapler. 
patients. Twenty-six patients had a carcinoma of the distal oesophagus or proximal stomach, and four had a benign peptic stricture. Of the malignant tumours, histologically 14 were adenocarcinoma, 10 were squamous cell carcinoma, and two were undifferentiated carcinoma. Paraoesophageal or coeliac lymph nodes or both contained tumour in 14 patients. A left thoracoabdominal incision was used in 24 patients with distal disease, while an Ivor Lewis approach was adopted in six patients with mid-oesophageal lesions. A pyloroplasty was performed in 15 patients. There were 18 men and 12 women in the series and their ages ranged from 41 to 77 years.

\section{OPERATIVE PROCEDURE}

An initial diagnosis is made in all cases by means of barium studies and oesophagoscopy with biopsy. The site of the lesion dictates the surgical approach; middle third oesophageal lesions are resected using the Ivor Lewis technique while lower oesophageal and high gastric lesions are approached through a left thoracoabdominal incision dividing the costal margin. The diaphragm is incised circumferentially.

If the tumour is considered resectable the stomach is mobilised fully, preserving the right gastric and gastroepiploic vessels. Lymphatic tissue around the

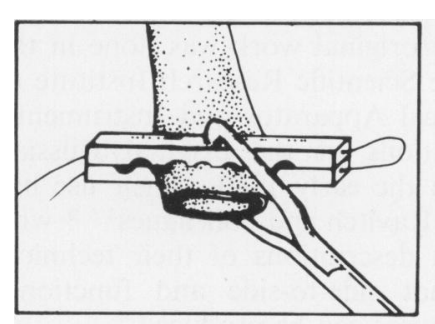

coeliac axis and along the superior border of the pancreas is excised en bloc with the left gastric artery, which is ligated and divided at its origin.

A pyloroplasty is performed routinely with all Ivor Lewis resections, but only in those lower third resections where the pylorus is narrowed, scarred, or contracted.

The site for gastric transection is selected and the stomach divided and closed using a stapling instrument (TA 90).

The point of oesophageal transection is chosen. This should be at least $4 \mathrm{~cm}$ above the uppermost limit of the lesion. A United States Surgical Pursestring instrument is placed across the oesophagus just proximal to the point of transection and a crushing clamp below. The oesophagus is transected and the specimen removed. A 1/0 Prolene suture on a straight needle is used to fashion a purse-string at the proximal end of the cut oesophagus (fig 2 inset). The purse-string instrument is removed and three stay-sutures inserted, two on the posterior wall and one on the anterior wall of the oesophagus, making sure that all layers are incorporated.

A longitudinal gastrotomy is made on the anterior wall of the stomach away from the staple line using the gastro-intestinal anastomosing device (GIA).
Fig 2 Use of purse-string instrument and introduction of stapling anvil into oesophagus. 
A stab incision is made and one fork of the GIA instrument is inserted in the lumen of the stomach, the instrument is closed and fired.

The loaded end-to-end anastomosing stapler (EEA)-without the anvil-is inserted through the anterior gastrotomy. A stab incision is made onto the rod of the instrument in the posterior wall of the stomach, 3-4 cms distal to the staple line, to ensure an adequate blood supply to the tissue between the staple lines and to avoid including any staples in the proposed site of anastomosis (fig 2). The stapling anvil is attached, lubricated, and then introduced into the oesophagus, the procedure being facilitated by means of the stay-sutures already in place. Great care is required at this stage to avoid tearing of the oesophagus. The purse-string suture is tied securely on to the rod. The EEA instrument is then closed so that the oesophageal and gastric parts of the anastomosis fit snugly between the cartridge and the anvil. Correct compression is achieved by aligning the vernier controls on the handles. The instrument is fired-a circular, staggered, double row of staples joins the organs in an end-to-side fashion. Simultaneously the circular blade in the instrument cuts the core of tissue within the rows of staples. The instrument is opened slightly and with a gentle rotatory movement it is removed. The staple line is inspected for haemostasis and to make sure that the mucosa is incorporated all the way round. A nasogastric tube is placed through the anastomosis from above and the gastrotomy is closed using a TA 55 stapling device.

The cartridge and anvil are examined for the circular tissue fragments (doughnuts). Two circumferential tissue fragments incorporating all layers indicate a well-fashioned anastomosis.

The gastric remnant is anchored to the hiatus using interrupted $3 / 0$ Mersilene sutures. The diaphragm is repaired and the incision closed in layers leaving one intercostal drain.

The patient is given nothing by mouth for four days. If there is no clinical evidence of an anastomotic leak clear fluids are allowed and the patient is advanced to a soft diet over a period of a few days. Antibiotics are given routinely and continued for five days after operation.

\section{Results}

There were two immediate postoperative deaths at three and four days respectively. Postmortem examination revealed evidence of septicaemia with bilateral adrenal haemorrhages in one and bilateral pleural effusions as well as ascites in the other. In both cases the anastomosis was intact and there was no evidence of any leak. A third patient developed a chylothorax which persisted. She required operative intervention and ligation of the thoracic duct six weeks after operation, but despite this she died from septicaemia and gross biochemical imbalance. Five patients developed significant atelectasis which responded to intensive chest physiotherapy. There were no anastomotic leaks, wound infections, or $\vec{\circ}$ thromboembolic episodes.

The EEA stapler failed to work satisfactorily in one patient. The staples were fired, but the knife did not cut the central core of tissue. This was noticed at the time of operation and an attempt was made to trim the excess tissues through the gastrotomy. After operation the patient complained of dysphagia when a more solid diet was instituted. Oesophagososcopy showed a pinhole opening with a rim of tissue within the row of staples. This was trimmed via the oesophagoscope using the biopsy forceps. She was swallowing normally when discharged from hospital. The reason for this malfunction appeared to be a failure of adequate lubrication of the instrument.

\section{Discussion}

Mechanical stapling devices for gastrointestinal and thoracic procedures have been in use since the early 1950s. Much of the original work was done in the Soviet Union at the Scientific Research Institute of Experimental Surgical Apparatus and Instruments. American modifications of the original Russian designs appeared in the early 1960s. Their use has been advocated by Ravitch and colleagues ${ }^{49}$ who have given detailed descriptions of their technical applications, so that side-to-side and functional end-to-side anastomoses can be produced.

In 1970 Androsov described the use of the Russian PKS stapler for performing circular anastomoses between the oesophagus and small bowel or stomach and between the colon and rectum. ${ }^{6}$ The PKS stapler fires a single row of staples and has to be loaded carefully. The United States Surgical o Corporation introduced the EEA stapler which is a $N$ modification of the original Russian instrument. N It fires a double, staggered row of staples which is more haemostatic and comes with a disposable anvil and staple cartridge which are easy to load. Its use in performing virtually all gastrointestinal anastomoses has been pioneered by Nance. ${ }^{7}$ More recently Dorsey et al ${ }^{10}$ have described its use in oesophagogastrectomy. West and his colleagues

report a similar experience. ${ }^{11}$
In a 10-year review of the literature Chassin ${ }^{12} \frac{\overrightarrow{\mathbb{D}}}{\square}$ found the average mortality for oesophagogastrectomy, in 13 reports, to be $20 \%$. About half of the 
overall mortality rate $(9 \%)$ was attributed to anastomotic leaks. End-to-side oesophagogastrectomy had a much lower incidence of fatal leaks (less than $2 \%$ ) when compared with end-to-end anastomoses.

We have described our technique for accomplishing an inverted, circular, end-to-side oesophagogastrectomy using the EEA stapler. The instrument is rather bulky and may be awkward to use in certain situations. However with practice the stapled anastomosis can be made faster than by conventional hand-sewn techniques. The anastomosis itself takes about 10 minutes to perform and the total operating time is also reduced. In our series the mean operating time was three hours for standard oesophagogastrectomies, and four hours five minutes for Ivor Lewis resections. The instrument allows less manipulation and therefore less trauma to the tissues than is involved with manual suturing. Soiling and bleeding are reduced with resultant faster healing. This allowed all our patients to be discharged home 11 days after operation. We have used all three sizes of head available $(25 \mathrm{~mm}$, $28 \mathrm{~mm}$, and $31 \mathrm{~mm}$ ) in the various situations and only had to abandon the use of the stapler on one occasion where the $25 \mathrm{~mm}$ head was still too large. Malfunctions similar to the one we have reported should not be a problem. The EEA is a precision instrument and therefore deserves scrupulous attention particularly to lubrication.

The initial financial outlay is quite considerable and the cartridges are also costly. However, this economic disadvantage is more than offset by the low incidence of complications, particularly anasto- motic leaks, with their resultant protracted and financially very costly management.

Our experience suggests that the EEA stapler creates a quick, wide, and secure anastomosis and represents a significant advance in oesophageal resection techniques.

\section{References}

${ }^{1}$ Gritsman JJ. Mechanical suture by Soviet apparatus in gastric resections: use in 4000 operations. Surgery 1966; 59:6639.

${ }^{2}$ Latimer RG, Doane WA, McKittrick D, Shepherd A. Automatic staple suturing for gastrointestinal surgery. Am J Surg 1975;130:766-71.

${ }^{3}$ Lawson WR, Hutchison J, Lougland CT, Hague MA. Mechanical suture methods in thoracic and abdominal Surgery. Br J Surg 1977;64:115-9.

${ }^{4}$ Ravitch MM, Rivarola A. Enteroanastomosis with an automatic stapling instrument. Surgery 1966;59:270-7.

${ }^{5}$ Ravitch MM, Lane R, Cornell WP, Rivarola A, McEnany T. Closure of duodenal, gastric and intestinal stumps with wire staples. Ann Surg 1966;163:573-9.

${ }^{6}$ Androsov PJ. Experience in the application of the instrumental mechanical suture in surgery of the stomach and rectum. Acta Chir Scand 1970;136:57-63.

${ }^{7}$ Nance FC. New techniques of gastrointestinal anastomoses with the EEA stapler. Ann Surg 1979;189:587-600.

${ }^{8}$ Ravitch MM, Steichen FM. A stapling instrument for end-to-end inverting anastomosis in the gastrointestinal tract. Ann Surg 1979;189:791-8.

${ }^{9}$ Ravitch MM, Steichen FM. Technics of staple suturing in the gastrointestinal tract. Ann Surg 1972;175:815-37.

${ }^{10}$ Dorsey JS, Esses S, Goldberg M, Stone R. Esophagogastrectomy using the auto suture EEA surgical stapling instrument. Ann Thorac Surg 1980;30:308-12.

11 West PN, Marbarger JP, Martz MN, Roper CL. Esophagogastrostomy with the EEA stapler. Ann Surg 1981; 193:76-81.

12 Chassin JL. Esophagogastrectomy: data favoring end-toside anastomosis. Ann Surg 1978;188:22-7. 